\title{
Biologically predisposed learning and selective associations in amygdalar neurons
}

\author{
Ain Chung, ${ }^{1}$ Sabiha K. Barot, ${ }^{2}$ Jeansok J. Kim, ${ }^{1,2}$ and Ilene L. Bernstein ${ }^{1,2,3}$ \\ ${ }^{1}$ Department of Psychology, University of Washington, Seattle, Washington 98195-1525, USA; ${ }^{2}$ Program in Neurobiology \& Behavior, \\ University of Washington, Seattle, Washington 98195-1525, USA
}

\begin{abstract}
Modern views on learning and memory accept the notion of biological constraints-that the formation of association is not uniform across all stimuli. Yet cellular evidence of the encoding of selective associations is lacking. Here, conditioned stimuli (CSs) and unconditioned stimuli (USs) commonly employed in two basic associative learning paradigms, fear conditioning and taste aversion conditioning, were delivered in a manner compatible with a functional cellular imaging technique (Arc cellular compartmental analysis of temporal gene transcription by fluorescence in situ hybridization [catFISH]) to identify biological constraints on CS-US convergence at the level of neurons in basolateral amygdala (BLA). Results indicate coincident Arc mRNA activation within BLA neurons after CS-US combinations that yield rapid, efficient learning, but not after CS-US combinations that do not.
\end{abstract}

[Supplemental material is available for this article.]

Early theories of learning and memory, grounded on the philosophy of associationism (Locke 1690), and empirical work in classical conditioning (Watson 1913; Pavlov 1927; Guthrie 1952), assumed that any stimulus could be associated with another stimulus with equal ease. This notion of equipotentiality in associative learning was effectively abandoned with the publication of several key reports (Breland and Breland 1961; Garcia and Koelling 1966; Garcia et al. 1974), which made it evident that some stimulus pairs formed associations with ease, whereas others developed slowly (or not at all). For example, tastes as conditioned stimuli (CSs) were readily associated with unconditioned stimuli (USs) (e.g., $\mathrm{LiCl}$ ) that induce gastrointestinal malaise leading to the emergence of learned taste aversions, whereas the very same taste CSs were ineffective cues for signaling shock USs. Conversely, a light/noise CS readily associated with a shock US to produce conditioned fear responses, but not with a LiCl US. Behavioral evidence of biologically predisposed associations continues to accumulate along with consideration of the adaptive function of selective associations (Hinde and Stevenson-Hinde 1973; Domjan 2005). What has been missing from this analysis is evidence of the neural and cellular mechanisms, which predispose some associations and not others.

It is widely assumed that the association of CS and US occurs at brain sites where information about the two converge (Thompson 1986; Abrams et al. 1991; Fanselow and Poulos 2005). In both fear and taste aversion conditioning, the basolateral nucleus of amygdala (BLA) appears to be a critical locus of CS-US association (Davis 1997; Fendt and Fanselow 1999; LeDoux 2000; Kim and Jung 2006; Barot et al. 2008, 2009). Despite the possibility of anatomical overlap, effective CSs in fear conditioning (context, tone) and taste aversion learning (novel tastes) show little efficacy when utilized in the alternative conditioning paradigm. Here we used Arc cellular compartmental analysis of temporal gene transcription by fluorescence in situ hybridization (catFISH) (Guzowski et al. 1999), to identify

${ }^{3}$ Corresponding author.

E-mail ileneb@u.washington.edu; fax (206) 685-3157.

Article is online at http://www.learnmem.org/cgi/doi/10.1101//m.2053711. populations of neurons displaying Arc (or Arg3.1) activation during single trial associative conditioning in rats to determine whether biologically predisposed associations yield evidence of convergence onto individual BLA neurons, whereas stimulus pairings that are ineffective do not.

Experimentally naive adult male Sprague Dawley rats (initially weighing 300-350 g) were individually housed and maintained on a reverse 12-h light/dark cycle (lights on at 19:00 h) with ad libitum access to food and water, except during the experiment. All experiments were conducted during the dark phase of the cycle and in accordance with guidelines approved by the Institutional Animal Care and Use Committee at the University of Washington.

Two separate studies (behavioral and catFISH) were run. In each, animals were randomly assigned to groups $(n=6$ rats per group): novel taste CS ( $0.5 \%$ saccharin solution) paired with $\mathrm{LiCl}$ US (0.15 M, $15 \mathrm{~mL} / \mathrm{kg}$ body weight) (T-L group); novel taste CS paired with footshock US ( $2 \mathrm{~mA}, 5 \mathrm{sec}$ ) (T-S group); novel context CS paired with LiCl US (C-L group); and novel context CS paired with footshock US (C-S group) (Fig. 1A). The C-S and T-S groups were conditioned in a modular operant test chamber $(27 \mathrm{~cm} \times 28 \mathrm{~cm} \times 30.5 \mathrm{~cm}$; wiped with $5 \%$ ammonium hydroxide solution; Coulbourn Instruments) located in a sound attenuating chamber. The grid floor was composed of 16 stainless steel bars (4.5 $\mathrm{mm}$ diameter) spaced $17.5 \mathrm{~mm}$ center to center and wired to a Coulbourn precision-regulated animal shocker. The T-L and C-L groups were conditioned in a different Coulbourn chamber (octagonal: $26.5 \mathrm{~cm}$ diameter $\times 25 \mathrm{~cm}$ height; wiped with $5 \%$ ammonium hydroxide solution) in a separate sound chamber.

Animals receiving novel taste as a CS (regardless of US type) were pre-exposed to the chamber for $10 \mathrm{~d}$ to reduce the salience of the context that interferes with appetitive behavior (Barot et al. 2009). Beginning on day 5 of pre-exposure and for subsequent days, animals were placed on water restriction to promote prompt saccharin intake during the $5 \mathrm{~min}$ of saccharin availability during training (day 11) and testing (day 12). During training, after $5 \mathrm{~min}$ of saccharin consumption, the solution bottle was removed, and after $20 \mathrm{~min}$, animals received either an i.p. injection of $\mathrm{LiCl}$ or a footshock. After an additional $5 \mathrm{~min}$ in the 


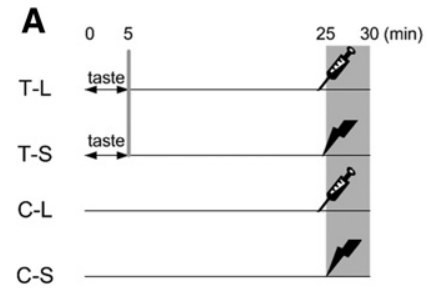

C-S
B

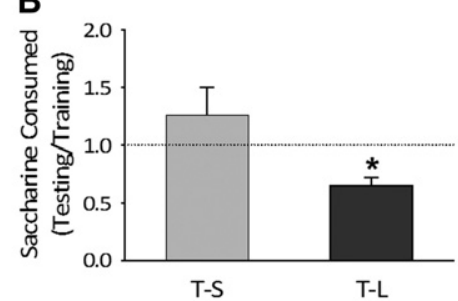

C

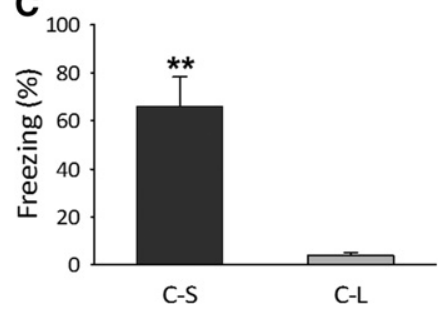

Figure 1. Experimental designs and results. $(A)$ A schematic timeline outlining presentation of the CS-US pairing for taste-LiCl (T-L), taste-shock (T-S), context- $\mathrm{LiCl}(\mathrm{C}-\mathrm{L})$, and context-shock (C-S) groups during training. (B) Conditioned taste aversion retention test the next test day. The T- $\mathrm{L}$ group showed reductions in saccharin consumption from training to test and differed significantly from the T-S group ( $t$-test, $* P<0.05$ ). ( $C$ ) Contextual fear retention test the next test day. The C-S group showed significantly higher levels of freezing behavior compared to the C-L group ( $t$-test, $* * P<0.005)$. Data are presented as mean \pm SEM.

chamber, they were either placed back in their home cage (behavioral groups) or sacrificed immediately (catFISH groups). Animals presented with novel context as a CS received either a footshock or $\mathrm{LiCl}$ injection $25 \mathrm{~min}$ after introduction into the chamber and remained in the chamber for an additional $5 \mathrm{~min}$ before either being placed back in their home cage (behavioral groups) or sacrificed immediately (catFISH groups). The memory retention tests (behavioral groups) were carried out $24 \mathrm{~h}$ after training. The taste CS animals were placed back in the chamber and permitted to drink saccharin solution for $5 \mathrm{~min}$. Saccharin intake was determined by measuring the solution bottle weight before and after $5 \mathrm{~min}$. The context CS animals were placed back in the chamber for $8 \mathrm{~min}$; freezing behavior was measured via a 24-cell infrared activity monitor mounted on top of the chamber and connected to the Coulbourn Instrument LabLinc Habitest Universal Linc System (cf. Lee and Kim 1998). Final group sizes were reduced by the exclusion of one animal in the behavioral study and six animals in the catFISH study. This was due to tissue damage, which interfered with scoring of tissue or behavioral anomalies including failure to freeze during testing (freezing score $>3 \mathrm{SD}$ from the rest of the group) or jumping out of the cage. Final $n$ 's in the groups in the catFISH study were $\mathrm{T}-\mathrm{L}=4, \mathrm{~T}-\mathrm{S}=5, \mathrm{C}-\mathrm{L}=$ 4 , and $\mathrm{C}-\mathrm{S}=5$.

Animals used for catFISH analysis in the BLA (Fig. 2) were exposed to the same CS-US conditioning experiences outlined previously, but were sacrificed $5 \mathrm{~min}$ after $\mathrm{LiCl}$ or footshock administration to allow US-induced Arc mRNA expression (Barot et al. 2008, 2009). Prior work using catFISH analysis and these conditioning paradigms established that little or no Arc mRNA expression is seen in BLA in control groups; namely groups exposed to familiar tastes or contexts, "no shock" or injected with saline rather than $\mathrm{LiCl}$ (Barot et al. 2008, 2009).

In the present experiment, animals in the catFISH study exhibited comparable inactivity and freezing during training as those used in behavioral experiments (Supplemental Fig. S1). Immediately after training, brains were rapidly extracted $(<1 \mathrm{~min})$, fresh frozen, and stored at $-80^{\circ} \mathrm{C}$. Forebrain tissue was sectioned into $20-\mu \mathrm{m}$ coronal slices using a cryostat and mounted onto slides. The BLA region (anteroposterior, $-2.7 \pm$ $0.1 \mathrm{~mm}$ from bregma) was selected for in situ hybridization. Digoxigenin-labeled Arc riboprobes were generated from a modified cDNA plasmid (provided by P. Worley, Johns Hopkins University School of Medicine) and fluorescent in situ hybridization for Arc was carried out using a standard procedure (Guzowski et al. 1999; Barot et al. 2008). Arc signal was tagged using the Cyanine 3 TSA system (Perkin-Elmer); nuclei were counterstained using Sytox Green (Invitrogen). A corresponding region of BLA was analyzed per rat in the manner described previously (cf. Barot et al. 2008, 2009). The experimenter was blind to group membership during image capture and data analysis. For each BLA, $211-\mu \mathrm{m}$-thick optical section images were acquired using a Leica SL microscope with a $20 \times$ objective lens using $\mathrm{GrHe} / \mathrm{Ne}$ and argon lasers. Z-series stacks were combined and analyzed on the MetaMorph 6.0 program. Manual counting of Arc positive cells used the following standard: (1) Careful optical dissection was done to ensure that only neurons with fully intact nuclei were scored; (2) nuclei had to be present in the median plane of the Z-stack and fully present throughout the stack without damage to be scored; and (3) neurons were scored as being positive for cytoplasmic staining if a "halo" of signal was found to be encircling at least $75 \%$ of the nucleus. For neurons to be scored as positive for nuclear staining, robust foci with high saturation $-\geq 230$ luminescent on the red channel of the MetaMorph programwere required.

Freezing, saccharin volume consumption, and the number of Arc + cells were analyzed via one-way ANOVA and independent $t$-test (SPSS 11).

When memory retention was assessed the day following conditioning (in the behavioral study), rats demonstrated that they associated the sweet taste CS with $\mathrm{LiCl}$ but not with footshock (Fig. 1B). Specifically, the saccharin consumption of taste- $\mathrm{LiCl}$ paired animals was significantly lower (evidence of conditioned taste aversion) than saccharin consumption of taste-footshock paired animals during $5 \mathrm{~min}$ in the test chamber $\left(t_{(10)}=2.48\right.$; $P<0.05)$. When context-conditioned animals were placed back in the conditioning chamber for an 8-min retention test, the context-footshock paired animals displayed robust conditioned
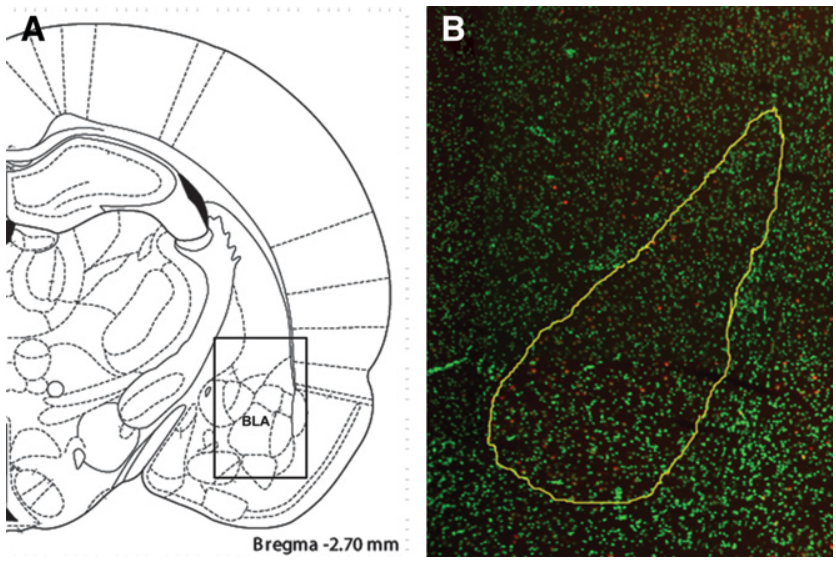

Figure 2. A representative image $(4 \times)$ of the BLA displaying Arc + staining (in red, nuclei are counterstained green). 
freezing, whereas the context-LiCl paired animals did not $\left(t_{(9)}=\right.$ 5.11; $P<0.01$ ) (Fig. 1C). These behavioral data indicate that rats are biologically predisposed to promptly learn saccharin-LiCl and context-footshock associations, but not saccharin-footshock and context- $\mathrm{LiCl}$ associations, confirming and extending the original findings of Garcia and Koelling (1966) and Garcia et al. (1974), and demonstrating that robust conditioned taste aversion and contextual fear conditioning can be acquired in a single trial using CS-US interstimulus interval (ISI) parameters that temporally correspond with catFISH analysis.

The saccharin CS- and context CS-induced Arc mRNA expressions localized to the cytoplasm of BLA neurons since the CS was presented $30 \mathrm{~min}$ before sacrifice (Fig. 3A). No significant differences were seen in the number of BLA neurons showing cytoplasmic Arc signal within each CS type: saccharin-LiCl vs. saccharin-footshock, and context-LiCl vs. context-footshock (Fig. 3B,C), although there was a trend toward greater responses in the effective conditioning (T-L and C-S) groups. Given that the US was delivered $5 \mathrm{~min}$ before sacrifice, $\mathrm{LiCl}$ and footshock exposure induced a punctate Arc mRNA signal that was confined to the nuclei of BLA neurons (Fig. 3A). Our interpretation that the $\mathrm{LiCl}$, and not the injection per se, was responsible for nuclear Arc is based on our prior work with identical procedures (Barot et al. 2008), which demonstrated that, unlike LiCl injections, saline injections do not promote Arc mRNA in BLA neurons.

Importantly, the number of BLA neurons displaying dual cytoplasmic and nuclear expression of Arc mRNA was reliably greater after pairings that yield learning (saccharin-LiCl $\left[t_{(7)}=\right.$ 2.67, $P<0.05]$ and context-footshock $\left.\left[t_{(7)}=2.67, P<0.05\right]\right)$ than after pairings that fail to yield learning (saccharin-footshock and context-LiCl) (Fig. 3B,C). Thus, for a given CS, dual Arc expression, indicative of convergent activation, was selective for the type of US that readily associated with it. This pattern was

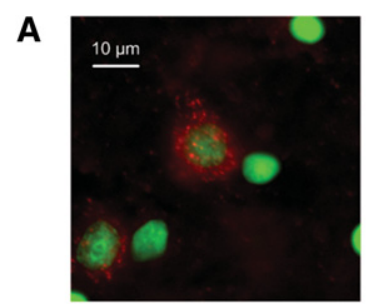

B

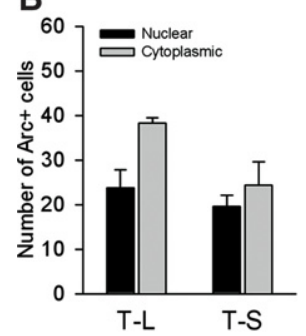

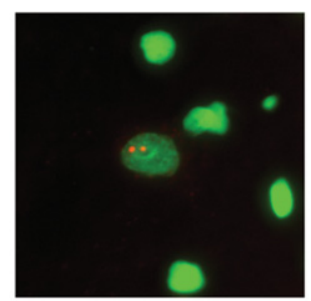
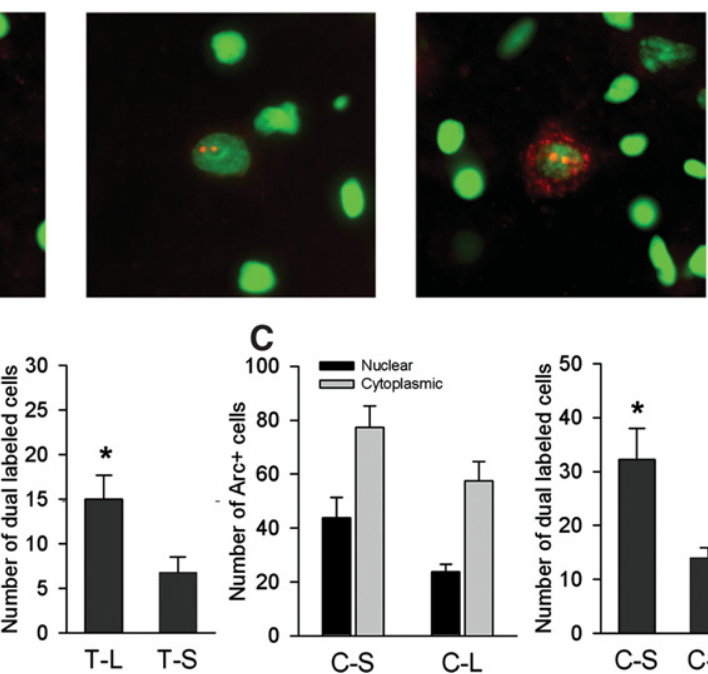

${ }_{100}^{C}$

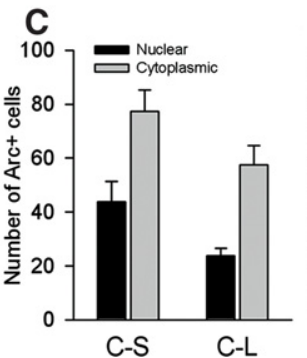

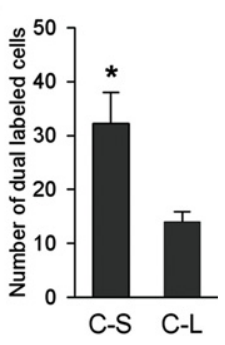

Figure 3. Arc localization in BLA with different CS-US pairings. ( $A$ ) Representative images $(40 \times)$ from BLA showing Arc localization following CS-US presentations. Neurons responding only to the earlier CS event show Arc signal (in red) in the cytoplasm surrounding the nucleus (counterstained green) (left panel); neurons responding only to the later US event show dense Arc foci confined to the nucleus (middle panel); neurons responding to both the CS and US show cytoplasmic and nuclear staining (right panel). (B) (Left) Number of neurons showing Arc + staining in either the nucleus or cytoplasm in T-L $(n=4$ animals) and T-S $(n=5)$ groups. (Right) Total number of cells in the BLA with dual Arc + activation, which is in both nuclear and cytoplasmic compartments, for T-L and T-S groups. The T-L group showed significantly more dual ArC + activation than the T-S group ( $t$-test, $\left.{ }^{*} P<0.05\right)$. (C) (Left) Number of neurons showing Arc + staining in either the nucleus or cytoplasm in C-L $(n=4), \mathrm{C}-\mathrm{S}(n=5)$ groups. (Right) Total number of cells in the BLA with dual Arc + activation, which is in both nuclear and cytoplasmic compartments, for C-L and C-S groups. The C-L group showed significantly more dual Arc + activation than the C-S group (t-test, ${ }^{*} P<0.05$ ). Data are presented as mean \pm SEM. clearest with the saccharin CS, where the number of convergent cells was extremely low in the group that failed to learn. With the context CS, however, there was some discrepancy between text-LiCl paired animals exhibited no evidence of fear learning, some BLA cells did exhibit dual Arc + staining. The implication of these findings is that a novel context may weakly associate where is, in fact, evidence that contexts paired response is a conditioned place aversion (CPA) not conditioned fear (White and Carr 1985). Indeed we found that a mild CPA to the context could occur in a single trial using the temporal parameters employed in these conditioning studies (Supplemenal Fig. S2).

Despite the long-standing behavioral evidence for selective, catFISH analysis provides the first molecular correlate for this phenomenon at the cellular level. The stimuli (CSs and USs) and temproting learning; but their effectiveness was selective, dependgent neuronal activation pairings (taste-LiCl; context-shock), but not after ineffective pairings (taste-shock). The demonstration of convergence of CS and US information onto individual BLA neurons replicates ditioned taste aversion (Barot et al. 2008, 2009). However, the present findings demonstrate that such coincident activation is and predictive as to whether a given pairing will be effective in promoting learning.

A caveat must be considered given that two different types of CSs were used. It is possible that $5 \mathrm{~min}$ of saccharin CS followed 20 min later with a footshock US resulted in poor trace pairing. Based on John Garcia and colleagues' extensive studies (e.g., Garcia and Koelling 1966; Garcia et al. 1974), where this trace distinction was not present and yet associations were highly selective, it is unlikely that reduced taste-shock association is simply due to trace pairing. Also, in Barot et al. (2009), 30-min placement in the context CS produced Arc + cells transiently (in both BLA and hippocampus), that is, only during the initial $5 \mathrm{~min}$, suggesting that in this sense our context-shock procedure (with a long 25-min ISI) could also be considered trace pairing.

It is likely that biologically predisposed associations can be based on a range of circuit and cellular properties, depending on the nature and modality of the stimuli involved. In the simplest cases, stimulus convergence may be precluded by information streams that show little or no anatomical overlap. A more challenging case is encountered when a single brain area, such as basolateral amygdala, is known to be a site of CS-US convergence for two different learning paradigms (Barot et al. 2008, 2009). The results presented here deal with this more complex situation and show that, although CS-US convergence 
for CTA and fear learning occur in amygdalar neurons, significant numbers of convergent cells are only seen with stimulus pairings that yield prompt learning. Thus, it appears that selective associations depend on highly specific interactions at the cellular and molecular level, which facilitate coincident activations for biologically predisposed stimulus pairs while precluding others. Perhaps, such highly selective information processing is essential for the diversity of functions in structures like the amygdala. Thus, specific microcircuits and cell signaling dynamics ensure that associations that are likely to provide significant survival benefits activate neurons in a convergent fashion and generate prompt learning. Such signaling dynamics are not recruited for stimulus associations with little likelihood of aiding an organism's fitness.

\section{Acknowledgments}

This work was supported by NIH grant R01 NS37040, a Royalty Research Grant from the University of Washington (I.L.B.), and a Cattell Sabbatical Award and R01 MH64457 (J.J.K.).

\section{References}

Abrams TW, Karl KA, Kandel ER. 1991. Biochemical studies of stimulus convergence during classical conditioning in Aplysia: dual regulation of adenylate cyclase by $\mathrm{Ca} 2+/$ calmodulin and transmitter. J Neurosci 11: $2655-2665$.

Barot SK, Kyono Y, Clark EW, Bernstein IL. 2008. Visualizing stimulus convergence in amygdala neurons during associative learning. Proc Natl Acad Sci 105: 20959-20963.

Barot SK, Chung A, Kim JJ, Bernstein IL. 2009. Functional imaging of stimulus convergence in amygdalar neurons during Pavlovian fear conditioning. PLoS ONE 4: e6156. doi: 10.1371/journal.pone.0006156.

Breland K, Breland M. 1961. The misbehavior of organisms. Amer Psychologist 16: 681-684.
Davis MJ. 1997. Neurobiology of fear responses: The role of the amygdala. Neuropsychiatry Clin Neurosci 9: 382-402.

Domjan M. 2005. Pavlovian conditioning: A functional perspective. Annu Rev Psychol 56: 179-206.

Fanselow MS, Poulos AM. 2005. The neuroscience of mammalian associative learning. Annu Rev Psychol 56: 207-234.

Fendt M, Fanselow MS. 1999. The neuroanatomical and neurochemical basis of conditioned fear. Neurosci Biobehav Rev 23: 743-760.

Garcia J, Koelling RA. 1966. Relation of cue to consequence in avoidance learning. Science 4: $123-124$.

Garcia J, Hankins WG, Rusiniak KW. 1974. Behavioral regulation of the milieu interne in man and rat. Science 185: 824-831.

Guthrie ER. 1952. The psychology of learning. Harper and Row, New York.

Guzowski JF, McNaughton BL, Barnes CA, Worley PF. 1999. Environment-specific expression of the immediate-early gene Arc in hippocampal neuronal ensembles. Nat Neurosci 2: 1120-1124.

Hinde RA, Stevenson-Hinde J. 1973. Constraints on learning. Academic, London, UK.

Kim JJ, Jung MW. 2006. Neural circuits and mechanisms involved in Pavlovian fear conditioning: A critical review. Neurosci Biobehav Rev 30: $188-202$.

LeDoux JE. 2000. Emotion circuits in the brain. Annu Rev Neurosci 23: $155-184$.

Lee H, Kim JJ. 1998. Amygdalar NMDA receptors are critical for new fear learning in previously fear-conditioned rats. J Neurosci 18: $8444-8454$

Locke J. 1690. An essay concerning human understanding (ed. R Woolhouse), p. 306. Penguin Books, New York.

Pavlov IP. 1927. Conditioned reflexes. Oxford University Press, London, UK. Thompson RF. 1986. The neurobiology of learning and memory. Science 233: $941-947$.

Watson JB. 1913. Psychology as the behaviourist views it. Psych Rev 20: $158-177$.

White NM, Carr GD. 1985. The conditioned place preference is affected by two independent reinforcement processes. Pharmacol Biochem Behav 23: $37-42$.

Received October 26, 2010; accepted in revised form March 13, 2011. 


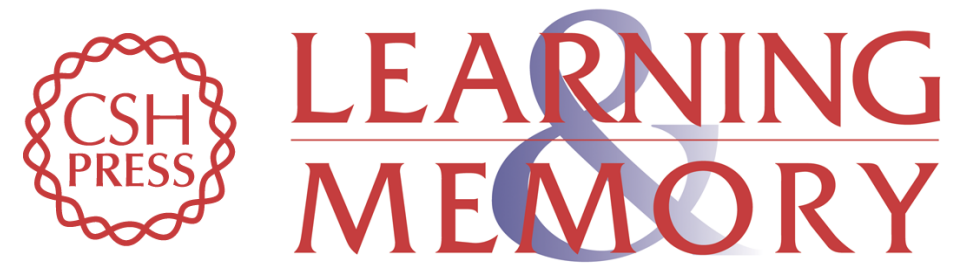

\section{Biologically predisposed learning and selective associations in amygdalar neurons}

Ain Chung, Sabiha K. Barot, Jeansok J. Kim, et al.

Learn. Mem. 2011, 18:

Access the most recent version at doi:10.1101/lm.2053711

Supplemental
Material http://learnmem.cshlp.org/content/suppl/2011/05/16/18.6.371.DC1

References This article cites 17 articles, 5 of which can be accessed free at:

http://learnmem.cshlp.org/content/18/6/371.full.html\#ref-list-1

License

Email Alerting Receive free email alerts when new articles cite this article - sign up in the box at the Service top right corner of the article or click here. 